\title{
REGULARITY FOR PARABOLIC SYSTEMS OF UHLENBECK TYPE WITH ORLICZ GROWTH
}

\author{
LARS DIENING, TONI SCHARLE, AND SEBASTIAN SCHWARZACHER
}

\begin{abstract}
We study the local regularity of $p$-caloric functions or more generally of $\varphi$-caloric functions. In particular, we study local solutions of non-linear parabolic systems with homogeneous right hand side, where the leading terms has Uhlenbeck structure of Orlicz type. This paper closes the gap of [22] where Liebermann proved that if the gradient of a solution is bounded, it is Hölder continuous.

The crucial step is a novel local estimates for the gradient of the solutions, which generalize and improve the pioneering estimates of DiBenedetto and Friedman [12, 10] for the $p$-Laplace heat equation.
\end{abstract}

Non-linear PDEs, Degenerate parabolic systems, Gradient estimates, De Giorgi technique. MSC: 35K40, 35B45, 35K65, 35B65.

\section{INTRODUCTION}

In this paper we study the local regularity of $p$-caloric functions and $\varphi$-caloric functions. The $p$-caloric functions are local, weak solutions of the $p$-Laplace heat equation

$$
\partial_{t} u-\operatorname{div}\left(|\nabla u|^{p-2} \nabla u\right)=0
$$

with $1<p<\infty$. The $\varphi$-caloric functions are local, weak solutions of the $\varphi$-Laplace heat equation

$$
\partial_{t} u-\operatorname{div}\left(\frac{\varphi^{\prime}(|\nabla u|)}{|\nabla u|} \nabla u\right)=0
$$

where $\varphi:[0, \infty) \rightarrow[0, \infty)$ is an Orlicz function satisfying the natural condition $\varphi^{\prime \prime}(t) t \approx \varphi^{\prime}(t)$, see Assumption 2.1 for more details. The case $\varphi(t)=\frac{1}{p} t^{p}$ corresponds to $p$-caloric functions, so $p$-caloric functions are a special case of $\varphi$-caloric functions. All solutions in this paper may be scalar or vectorial, i.e. we study both equations and systems.

More explicitly, let $J$ be a time interval and $\Omega$ a domain in $\mathbb{R}^{n}$. Then we will study local weak solutions $u$ of (1.2). In particular, we study functions $u: J \times \Omega \rightarrow \mathbb{R}^{N}$ with $u \in L^{\infty}\left(J, L^{2}(\Omega)\right)$ and $\varphi(|\nabla u|) \in L^{1}\left(J, L^{1}(\Omega)\right)$ such that

$$
\int \frac{\varphi^{\prime}(|\nabla u|)}{|\nabla u|} \nabla u \cdot \nabla \zeta \mathrm{d} z=\int u \cdot \partial_{t} \zeta \mathrm{d} z
$$

for all $\zeta \in C_{0}^{\infty}\left(J \times \Omega ; \mathbb{R}^{N}\right)$. Our main result is the local boundedness of the gradients $\nabla u$, see Theorem 2.2.

Let us begin with the case of $p$-caloric functions. It is known that if $\nabla u$ is locally in $L^{2}$, then $\nabla u$ is already Hölder continuous. This was proven in the celebrated works of DiBenedetto and Friedman 11 , 12. In the first step of the proof the authors show the boundedness of $\nabla u$. Unfortunately, it was necessary to have separate proofs for the sub-linear case $p \leq 2$ and the super-linear case $p \geq 2$. We will introduce in this paper a new approach that allows to handle both cases at once.

The origins to prove $L^{\infty}$ bounds of quasi-linear or non-linear parabolic solutions was achieved by Nash [26] and Moser 25] by the celebrated DiGiorgi-Nash-Moser technique. For degenerate non-linear elliptic equations this technique was adapted by Ural'ceva 29 and for non-linear elliptic systems by Uhlenbeck 28]. Both authors proved Hölder continuity of the gradients of $p$-harmonic functions, i.e. solutions of $\operatorname{div}\left(|\nabla u|^{p-2} \nabla u\right)=0$. Up to this day this is the best regularity result for homogeneous solutions to the p-Laplace equation which is known for space dimensions $n \geq 3$.

S. Schwarzacher thanks the project MORE LL1202 financed by the Ministry of Education, Youth and Sports, Czech Republic. 
Later it was observed by various authors, that the growth restrictions can be generalized to Orlicz growth. For the elliptic homogeneous theory we refer to [21, 23, 16, 8] and also the book [6]. Under natural assumptions on the Orlicz growth it is shown in these references, that the gradients of local weak solutions are Hölder continuous. As a consequence the so called non-linear Calderón-Zygmund theory was applicable and many results for elliptic systems with Orlicz growth and inhomogeneous right hand side were proven, see for example [14, 17, 2].

Let us consider now the case of $\varphi$-caloric functions. Lieberman showed, that $\varphi$-caloric functions with bounded gradients already have Hölder continuous gradients. However, the step proving local boundedness of the gradients was still missing. For equations this gap was closed independently by Baroni and Lindfors [3, Theorem 1.2]. In this work we prove the boundedness of the gradients in to the general vectorial case.

The crucial step lies in a novel $L^{\infty}$-gradient estimate that quantitatively improves the known ones even for $p$-caloric functions, in particular those from the seminal work of DiBenedetto and Friedman 11, 12. In their work the gradients are pointwisely estimated in terms of the maximum of a constant and a suitable mean average of the gradients. Therefore, if the average of the gradients is small, the estimates of DiBenedetto and Friedman only imply that $|\nabla u| \leq c$. In contrast, in our estimate small averages imply small $L^{\infty}$ bounds. See the discussions after Remark 2.4 for more details.

Finally, our proof is a lot shorter than the original one by DiBenedetto and Friedman and allows to treat the entire range $p \in\left(\max \left\{1,2-\frac{4}{n}\right\}, \infty\right)$ in one step.

\section{Notations AND MAIN RESUlts}

To simplify the notation the letter $c$ will denote a positive constant, which may vary throughout the paper but will not depend on the important quantities. We write $f \lesssim g$ for $f \leq c g$ and $f \gtrsim g$ for $f \geq c g$. Moreover, we write $f \approx g$ if $f \lesssim g \lesssim f$. We say that a function $f:(0, \infty) \rightarrow(0, \infty)$ is almost increasing if $f\left(t_{2}\right) \leq c f\left(t_{1}\right)$ for all $t_{2} \geq t_{1}$. For a ball $B$, resp. cylinder $Q$, and $\lambda>0$ we define $\lambda B$, resp. $\lambda Q$, as the ball/cylinder with the same center but the radius scaled by the factor $\lambda$.

We begin, by introducing the assumptions on our Orlicz function $\varphi$. The assumptions are quite standard and motivated by the elliptic theory, see for example [16].

Assumption 2.1. Let $\varphi:[0, \infty) \rightarrow[0, \infty)$ be convex function with $\varphi \in C^{2}((0, \infty)) \cap C^{1}([0, \infty))$, $\varphi(0)=0, \varphi^{\prime}(0)=0$ and $\lim _{t \rightarrow \infty} \varphi(t)=\infty$. Moreover, we assume, that

$$
\varphi^{\prime}(t) \approx \varphi^{\prime \prime}(t) t
$$

The constants hidden in " $\sim$ will be referred to as the characteristics of $\varphi$.

One consequence of Assumption 2.1 is that $\varphi$ and its conjugate function $\varphi^{*}$, given by $\varphi^{*}(s)=$ $\sup _{t \geq 0}(s t-\varphi(t))$, automatically satisfies the $\Delta_{2}$ condition, i.e. $\varphi(2 t) \leq c \varphi(t)$ and $\varphi^{*}(2 t) \leq c \varphi^{*}(t)$ for all $t \geq 0$, see for example (2.2) of [4. This excludes linear and exponential growth. The functions $\varphi(t)=\frac{1}{p} t^{p}$ with $1<p<\infty$ satisfy Assumption 2.1

Our main theorem is the following

Theorem 2.2. Let $\varphi$ satisfy Assumption 2.1 and let $\rho(t):=(\varphi(t))^{\frac{n}{2}} t^{2-n}$ be almost increasing. Further, let $u$ be a local weak solution to (1.2) on $J \times \Omega$ with $\nabla u \in L_{\text {loc }}^{2}\left(J, L_{l o c}^{2}(\Omega)\right)$. Then for any cylinder $Q:=\left(t-\alpha r^{2}, t\right) \times B_{R}(x)$ with $2 Q \subset J \times \Omega$ we have

$$
\min \left\{\sup _{Q} \frac{\rho(|\nabla u|)}{\alpha^{\frac{2-n}{2}}}, \sup _{Q} \frac{|\nabla u|^{2}}{\alpha}\right\} \leq c \int_{2 Q} \frac{|\nabla u|^{2}}{\alpha}+\varphi(|\nabla u|) \mathrm{d} z .
$$

The constant only depends on the characteristics of $\varphi$.

The proof of this theorem can be found on 11 .

The assumption in Theorem 2.2 that $\rho$ is almost increasing reflects the usual restriction of the exponents near 1 for the parabolic p-Laplace. These restrictions arise due to the different scaling of the time derivative and the elliptic part. This effect is sometimes also called scaling deficit. It would be desirable in (2.2) to have $\rho(|\nabla u|) \alpha^{\frac{n-2}{2}}$ replaced by $\varphi(|\nabla u|)$. However, the scaling deficit prevents this. The only case with no scaling deficit, is $\varphi(t)=\frac{1}{2} t^{2}$, which corresponds to the standard heat equation. Only then, we have $\rho(t) \approx \varphi(t)$. See Remark 2.5 for an explanation, how the restriction $p \geq 2-\frac{4}{n}$ arises in the proof. 
Examples that satisfy the assumptions of Theorem 2.2 are $\varphi(t)=\frac{1}{p} t^{p}$, for $p \in\left(\max \left\{1,2-\frac{4}{n}\right\}, \infty\right)$. In this case (1.2) becomes the p-Laplace heat equation. Also $\varphi(t)=\max \left\{t^{p}, t^{q}\right\}$ or $\varphi(t)=\min \left\{t^{p}, t^{q}\right\}$ with $\max \left\{1,2-\frac{4}{n}\right\}<p \leq q<\infty$ satisfies the assumptions of Theorem 2.2

Since the case of $p$-caloric functions is of special interest, let us phrase our main result for this special situation.

Corollary 2.3. Let $p>2-\frac{4}{n}$ and let $u$ be a local weak solution to (1.1) on $J \times \Omega$ with $|\nabla u| \in$ $L_{\text {loc }}^{2}(J \times \Omega)$. Denote $\nu_{2}=n(p-2)+4$. For any cylinder $Q=\left(t-\alpha R^{2}, t\right) \times B_{R}(x)$ with $2 Q \subset J \times \Omega$ we have

$$
\min \left\{\sup _{Q} \frac{|\nabla u|^{\frac{\nu_{2}}{2}}}{\alpha^{\frac{2-n}{2}}}, \sup _{Q} \frac{|\nabla u|^{2}}{\alpha}\right\} \leq c \int_{2 Q} \frac{|\nabla u|^{2}}{\alpha}+|\nabla u|^{p} \mathrm{~d} z,
$$

where the constant $c$ only depends on $p, n$.

Remark 2.4. Note that in Theorem 2.2 as well as in Corollary 2.3 we need $\nabla u \in L_{\text {loc }}^{2}(2 Q)$. For p-caloric functions with $p \geq 2$, this regularity is natural. In the sub-linear case, this is not obvious. However, for the initial boundary value problem of $\varphi$-caloric functions on the whole space $\mathbb{R}^{n}$ with initial values in $W^{1,2}(\Omega)$, this regularity follows for example from [FS15, Theorem 5.1].

Let us compare this novel result to the estimates DiBenedetto 10, Chap VIII, Theorem 5.1 and Theorem 5.2]. There the following estimates are proven.

$$
\begin{array}{cl}
\sup _{Q} \frac{|\nabla u|^{2}}{\alpha} \leq \max \left\{c \int_{2 Q}|\nabla u|^{p} \mathrm{~d} z, \alpha^{\frac{p}{2-p}}\right\} & \text { for } p \geq 2, \\
\sup _{Q} \frac{|\nabla u|^{\frac{\nu_{2}}{2}}}{\alpha^{\frac{2-n}{2}}} \leq \max \left\{c \int_{2 Q} \frac{|\nabla u|^{2}}{\alpha} \mathrm{d} z, \alpha^{\frac{p}{2-p}}\right\} & \text { for } p \leq 2 .
\end{array}
$$

It follows from these estimates that

$$
\min \left\{\sup _{Q} \frac{|\nabla u|^{\frac{\nu_{2}}{2}}}{\alpha^{\frac{2-n}{2}}}, \sup _{Q} \frac{|\nabla u|^{2}}{\alpha}\right\} \leq \max \left\{c \int_{2 Q} \frac{|\nabla u|^{2}}{\alpha}+|\nabla u|^{p} \mathrm{~d} z, \alpha^{\frac{p}{2-p}}\right\} .
$$

To compare this estimates with (2.3) it is the easiest to think of the case $\alpha=1$. If the average integrals on the right hand side of (2.5) are small, then we get 1 on the right side. Thus, we get a local bound for the gradients, but do not know if the gradients must be small. The estimate (2.3) however allows to deduce smallness of the gradients if the average integrals are small. This is a novel improvement.

The proof of Theorem 2.2 is based on the DeGiorgi iteration technique. The necessary gain in integrability is achieved by the following theorem, which is proved in Section 3 and is of independent interest. Note that the quantity $V(\nabla u)$ in this theorem is a very natural quantity for equations involving the $p$-Laplacian, resp. $\varphi$-Laplacian.

Remark 2.5. The condition $p>2-\frac{4}{n}$ is basically due to Theorem 3.4. It follows from this theorem and the embedding $W^{1,2} \hookrightarrow L^{\frac{2 n}{n-2}}$ applied to $V(\nabla u)$ that

$$
\nabla u \in L_{\mathrm{loc}}^{\infty}\left(J, L_{\mathrm{loc}}^{2}(\Omega)\right) \cap L_{\mathrm{loc}}^{p}\left(J, L_{\mathrm{loc}}^{\frac{p n}{n-2}}(\Omega)\right) .
$$

Now the condition $p>2-\frac{4}{n}$ is equivalent to the parabolic embedding

$$
L_{\mathrm{loc}}^{\infty}\left(J, L_{\mathrm{loc}}^{2}(\Omega)\right) \cap L_{\mathrm{loc}}^{p}\left(J, L_{\mathrm{loc}}^{\frac{p n}{n-2}}(\Omega)\right) \hookrightarrow L_{\mathrm{loc}}^{2}\left(J, L_{\mathrm{loc}}^{2}(\Omega)\right) .
$$

We have shown in Theorem 2.2 the local boundedness of the gradients. We want to apply the results of Lieberman [22, Corollary 2.1] to obtain Hölder continuity of the gradients. Lieberman studied weak solutions of (1.2)

with certain conditions on $F$. In our situation we have

$$
\partial_{t} u-\operatorname{div}(F(|\nabla u|) \nabla u)=0
$$

$$
F(t)=\frac{\varphi^{\prime}(t)}{t} \quad \text { and } \quad \frac{t F^{\prime}(t)}{F(t)}=\frac{\varphi^{\prime \prime}(t) t}{\varphi^{\prime}(t)}-1 .
$$


Therefore, condition (1.2) of [22], namely $\delta-1 \leq \frac{t F^{\prime}(t)}{F(t)} \leq g_{0}-1$ for some $\delta, g_{0}>0$ is equivalent to our condition (2.1). Also conditions (2.2a) is a consequence of our condition (2.1), see Section 3 . However, to apply the result of Lieberman, we need to assume the following off-diagonal uniform continuity of $\varphi^{\prime \prime}$.

Assumption 2.6. Let $\varphi$ satisfy Assumption 2.1. Further assume that there exists a continuous function $\omega:\left(0, \frac{1}{2}\right) \rightarrow \mathbb{R}$ with $\omega(0)=0$ and

$$
\left|\varphi^{\prime \prime}(s)-\varphi^{\prime \prime}(t)\right| \leq c \omega\left(\frac{|s-t|}{t}\right) \varphi^{\prime \prime}(t) \quad \text { for all } s, t \geq 0 \text { with }|s-t|<\frac{1}{2} t
$$

We are now able to apply the results of Lieberman to our setting.

Theorem 2.7 (Lieberman [22, Corollary 2.1]). Let $\varphi$ satisfy Assumption[2.6. If $u$ is a weak solution to (1.2) on $J \times \Omega$, with $\nabla u \in L_{\text {loc }}^{2}\left(J, L_{\text {loc }}^{2}(\Omega)\right)$, then $\nabla u$ is locally Hölder continuous in $J \times \Omega$. Moreover, there exists a $\mu \in(0,1)$ such that for any cylinder $Q_{R}=\left(t-R^{2}, t\right) \times B_{R}(x) \subset J \times \Omega$ and $Q_{R}=\left(t-r^{2}, t\right) \times B_{r}(x)$ with $r<\frac{1}{2} R$ the following estimate is satisfied:

$$
\sup _{z, z_{2} \in Q_{r}}\left|\nabla u(z)-\nabla u\left(z_{2}\right)\right| \leq c \sup _{Q_{R}}|\nabla u| \cdot\left(\frac{r}{R} \kappa\left(\sup _{Q_{R}}|\nabla u|\right)\right)^{\mu}
$$

with $\kappa(t):=\max \left\{\sqrt{\varphi^{\prime \prime}(t)}, \sqrt{1 / \varphi^{\prime \prime}(t)}\right\}$ where $c$ depends on the characteristics of $\varphi$ and $\omega$ from Assumption 2.6 .

This theorem in combination with our Theorem 2.2 implies the local Hölder continuity of $\varphi$-caloric functions.

At this point we wish to emphasize the importance of the regularity results of Theorem 2.2 and Theorem 2.7 for systems with inhomogeneous right hand side. Regularity theory for non-linear PDE with inhomogeneous right hand side is successfully achieved by the so called non-linear Calderón Zygmund theory. It was first used for the $p$-Laplacian by Iwaniec [18, see also 9]. The core of this theory is the combination of a Calderón-Zygmund decomposition (e.g. of the gradient of the solution) combined with local comparison with $p$-harmonic, resp. p-caloric functions. The local regularity of the homogeneous system is therefore of fundamental importance. The local $L^{\infty}$-bound (of DiBenedetto and Friedman) of the gradient of $p$-caloric functions was successfully used to derive higher integrability results 1]. The Hölder estimates for the gradients of $p$-caloric functions has been used to show Hölder continuity for the inhomogeneous system [12, 24] and to derive estimates of BMO-Type [27]. Moreover, it is a necessary tool for the proof of pointwise potential estimates [19, 20] and for almost everywhere regularity results by p-caloric approximation [7].

\section{DifFEREntiability}

In this section we prove the higher regularity estimates of Theorem 3.5 . For this we have to start with a few properties on our N-function $\varphi$ and its relations to the quantities of our equation. Again we assume that $\varphi$ satisfies Assumption 2.1.

First of all, we define the quantities $A, V: \mathbb{R}^{n \times N} \rightarrow \mathbb{R}^{n \times N}$ by

$$
\begin{aligned}
A(P) & :=\varphi^{\prime}(|P|) \frac{P}{|P|}, \\
V(P) & :=\sqrt{\varphi^{\prime}(|P|)|P|} \frac{P}{|P|} .
\end{aligned}
$$

Then our $\varphi$-Laplacian heat equation can be written as

$$
\partial_{t} u-\operatorname{div}(A(\nabla u))=0 .
$$

The quantity $V(\nabla u)$ is well known from the regularity theory of systems with a $p$-Laplace, in which case $V(P)=|P|^{\frac{p-2}{2}} P$.

Moreover, we need the shifted $N$-function $\varphi_{a}$ from [13], which are defined for $t, a \geq 0$ as

$$
\left(\varphi_{a}\right)^{\prime}(t):=\frac{\varphi^{\prime}(a+t)}{a+t} t
$$


We have $\left(\varphi_{a}\right)_{b}=\varphi_{a+b}$. Note that the family $\varphi_{a}$ also satisfies Assumption 2.1 with characteristics uniformly bounded with respect to $a \geq 0$. This implies that the families $\varphi_{a}$ and $\left(\varphi_{a}\right)^{*}$ satisfy the $\Delta_{2}$-condition with constants independent of $a \geq 0$. Note that $\varphi_{0}=\varphi$. Moreover, uniformly in $a, t \geq 0$

$$
\begin{aligned}
\left(\varphi_{a}\right)(t) & \approx \varphi_{a}^{\prime}(t) t \approx \varphi_{a}^{\prime \prime}(t) t^{2} \approx \varphi^{\prime \prime}(a+t) t^{2}, \\
\left(\varphi_{a}\right)^{*}(t) & \approx\left(\varphi^{*}\right)_{\varphi^{\prime}(a)}(t) \approx\left(\varphi^{*}\right)^{\prime \prime}\left(\varphi^{\prime}(a)+t\right) t^{2} .
\end{aligned}
$$

The following lemma from 13 summarizes many important relations between $A, V$ and the shifted N-functions $\varphi_{a}$.

Lemma 3.1. Uniformly in $P, Q \in \mathbb{R}^{n \times N}$ we have

$$
\begin{gathered}
(A(P)-A(Q)):(P-Q) \\
\approx \varphi_{|P|}(|P-Q|) \approx|V(P)-V(Q)|^{2}, \\
|A(P)-A(Q)| \approx \varphi_{|P|}^{\prime}(|P-Q|) .
\end{gathered}
$$

In combination with (3.2) many variants of these equivalences follow.

We can apply the standard Young's inequality for N-function, which are in our context as follows: For every $\delta>0$ there exists $c_{\delta}>0$ such that uniformly in $a, s, t \geq 0$

$$
\begin{aligned}
& \varphi_{a}^{\prime}(s) t \leq \delta \varphi_{a}(s)+c_{\delta}\left(\varphi_{a}\right)^{*}(t), \\
& \varphi_{a}^{\prime}(s) t \leq c_{\delta} \varphi_{a}(s)+\delta\left(\varphi_{a}\right)^{*}(t) .
\end{aligned}
$$

Moreover, the following estimates are versions of Young's inequalities and can be found in [15].

Lemma 3.2 (Shift-change lemma). For every $\delta>0$ there exists $c_{\delta}>0$ such that for all $P, Q \in \mathbb{R}^{n \times N}$ and $t \geq 0$ we have

$$
\begin{aligned}
\varphi_{|P|}(t) & \leq c_{\delta} \varphi_{|Q|}(t)+\delta|V(P)-V(Q)|^{2} \\
\left(\varphi_{|P|}\right)^{*}(t) & \leq c_{\delta}\left(\varphi_{|Q|}\right)^{*}(t)+\delta|V(P)-V(Q)|^{2} .
\end{aligned}
$$

Moreover, we will need the following new estimate.

Lemma 3.3. There holds

$$
\left|\varphi_{a}^{\prime}(t)-\varphi^{\prime}(t)\right| \leq c \varphi_{t}^{\prime}(a)
$$

with $c$ independent of $a, t \geq 0$.

Proof. We have

$$
\left|\varphi_{a}^{\prime}(t)-\varphi^{\prime}(t)\right|=\left|\frac{\varphi^{\prime}(a+t)}{a+t} t-\varphi^{\prime}(t)\right| \leq \frac{t}{a+t}\left|\varphi^{\prime}(a+t)-\varphi^{\prime}(t)\right|+\frac{a}{a+t} \varphi^{\prime}(t)
$$

From (3.5) with $P=(a+t) M, Q=t M$ and $|M|=1$ it follows that $\left|\varphi^{\prime}(a+t)-\varphi^{\prime}(t)\right| \leq c \varphi_{t}^{\prime}(a)$, so

$$
\left|\varphi_{a}^{\prime}(t)-\varphi^{\prime}(t)\right| \leq c \frac{t}{a+t} \varphi_{t}^{\prime}(a)+\frac{a}{a+t} \varphi^{\prime}(a+t) \leq c \varphi_{t}^{\prime}(a) .
$$

The higher regularity of $\varphi$-caloric will be obtained by the difference quotient technique, which corresponds formally to a localized version of the testfunction $\Delta u$. Therefore we introduce the following notation: For a function $f: \mathbb{R}^{n} \rightarrow \mathbb{R}^{N}$ we write $\left(\tau_{h, j} f\right)(x)=f\left(x+h e_{j}\right)-f(x),\left(\delta_{h, j} f\right)(x)=$ $h^{-1}\left(f\left(x+h e_{j}\right)-f(x)\right)$ and $\delta_{h} f=\left(\delta_{h, 1} f, \ldots, \delta_{h, n} f\right)$. Moreover, we use the translation operator $T_{x}(y)=x+y$. The following theorem is a special case of Theorem 3.5 below with $f=1$.

Theorem 3.4. Let $\varphi$ satisfy Assumption 2.1 and let $u$ be a local weak solution to (1.2) on a cylindrical domain $J \times \Omega \subset \mathbb{R}^{1+n}$. For any cylinder $Q=\left(t-\alpha R^{2}, t\right) \times B_{R}(x) \Subset J \times \Omega$, we find:

If at the initial time $u\left(t-\alpha R^{2}\right) \in W^{1,2}(B)$, then for $\eta \in C_{0}^{\infty}(B)$ with $0 \leq \eta \leq 1$ we have

$$
\begin{aligned}
\sup _{I} & \frac{1}{\alpha R^{2}} \int_{B}|\nabla u|^{2} \eta^{q} \mathrm{~d} x+\int_{Q}|\nabla V(\nabla u)|^{2} \eta^{q} \mathrm{~d} z \\
& \lesssim f_{Q}|V(\nabla u)|^{2}\|\nabla \eta\|_{\infty}^{2} \mathrm{~d} z+\frac{1}{\alpha R^{2}} f_{B}\left|\nabla u\left(t-\alpha R^{2}\right)\right|^{2} \eta^{q} \mathrm{~d} z .
\end{aligned}
$$


If $\nabla u \in L_{\text {loc }}^{2}(J \times \Omega)$, then for $\eta \in C_{0}^{\infty}(Q)$ with $0 \leq \eta \leq 1$ we have

$$
\begin{aligned}
\sup _{I} & \frac{1}{\alpha R^{2}} \int_{B}|\nabla u|^{2} \eta^{q} \mathrm{~d} x+f_{Q}|\nabla V(\nabla u)|^{2} \eta^{q} \mathrm{~d} z \\
& \lesssim f_{Q}|V(\nabla u)|^{2}\|\nabla \eta\|_{\infty}^{2} \mathrm{~d} z+f_{Q}|\nabla u|^{2} \eta^{q-1}\left|\partial_{t} \eta\right| \mathrm{d} z .
\end{aligned}
$$

Here $q>1$ is fixed such that $\varphi_{a}\left(\eta^{q-1} t\right) \lesssim \eta^{q} \varphi_{a}(t)$, which exists due to [13, (6.25)].

Proof. The proof is based on the difference quotient technique and uses the test function $\xi=$ $\delta_{-h, j}\left(\eta^{q} \delta_{h, j} u\right)$. The proof is very similar to the one in [13, Theorem 11]. The terms involving $-\operatorname{div}\left(\varphi^{\prime}(|\nabla u|) \frac{\nabla u}{|\nabla u|}\right)$ are exactly as in [13. The terms involving the time derivatives are also quite standard to handle for a parabolic system. Indeed, they can be handled via a Steklov average as it was done in [10, Chap. VIII].

Theorem 3.5. Let $\varphi$ satisfy Assumption 2.1 and let $u$ be a local weak solution to (1.2) on a cylindrical domain $J \times \Omega \subset \mathbb{R}^{1+n}$ with $\nabla u \in L_{\text {loc }}^{2}(J \times \Omega)$. Further, let $f:[0, \infty) \rightarrow[0, \infty)$ be a non-decreasing function and define $H:[0, \infty) \rightarrow[0, \infty)$ by $H(0)=0$ and $H^{\prime}(t)=t f(t)$. For any cylinder $Q=$ $I \times B=\left(t-\alpha R^{2}, t\right) \times B_{R}(x) \Subset J \times \Omega$ and $\eta \in C_{0}^{\infty}(Q)$ with $0 \leq \eta \leq 1$ we have

$$
\begin{aligned}
\sup _{I} & \frac{1}{\alpha R^{2}} \int_{B} H(|\nabla u|) \eta^{q} \mathrm{~d} x+f_{Q}|\nabla V(\nabla u)|^{2} \eta^{q} f(|\nabla u|) \mathrm{d} z \\
& \lesssim f_{Q}|V(\nabla u)|^{2}\|\nabla \eta\|_{\infty}^{2} f(|\nabla u|) \mathrm{d} z+f_{Q} H(|\nabla u|) \eta^{q-1}\left|\partial_{t} \eta\right| \mathrm{d} z .
\end{aligned}
$$

At this $q>1$ is fixed such that $\varphi_{a}\left(\eta^{q-1} t\right) \lesssim \eta^{q} \varphi_{a}(t)$, which exists due to [13, (6.25)].

Proof. The proof is similar to Theorem 3.4 which is just a special case of this theorem with $f=1$. We already know from Theorem 3.4 that $\nabla V(\nabla u) \in L_{\mathrm{loc}}^{2}(J \times \Omega)$. In the following we abbreviate $v:=|\nabla u|$.

We now want to include the function $f$. By means of the monotone convergence theorem it suffices to prove the theorem under the additional assumption that $f \in C^{1}$ and that $f$ is constant for large values.

We take the test function $\xi=|Q|^{-1} \delta_{-h, j}\left(f\left(\left|\delta_{h} u\right|\right) \eta^{q} \delta_{h, j} u\right)$ over $j=\{1, \ldots, n\}$. With the standard treatment of the time derivative and $\partial_{t} H\left(\left|\delta_{h} u\right|\right)=\left(\partial_{t} \delta_{h} u\right) f\left(\left|\delta_{h} u\right|\right) \delta_{h} u$ one finds

$$
\begin{aligned}
\mathrm{I}+\mathrm{II} & :=\sum_{j} \int_{Q} \delta_{h, j} A(\nabla u) \cdot \nabla\left(f\left(\left|\delta_{h} u\right|\right) \eta^{q} \delta_{h, j} u\right) \mathrm{d} z+\frac{1}{\alpha R^{2}} \sup _{I} f_{B} H\left(\left|\delta_{h} u\right|\right) \eta^{q} \mathrm{~d} x \\
& \leq \int_{Q} H\left(\left|\delta_{h} u\right|\right)\left|\partial_{t}\left(\eta^{q}\right)\right| \mathrm{d} z=: \text { III. }
\end{aligned}
$$

Since we have $\nabla u \in L^{2}(Q)$ we have $\delta_{h, j} u \rightarrow \partial_{j} u$ in $L^{2}$. With this and $H(t) \leq \frac{\|f\|_{\infty}}{2} t^{2}$, we get as $h \rightarrow 0$

$$
\begin{gathered}
\mathrm{II} \rightarrow \frac{1}{\alpha R^{2}} \sup _{I} f_{B} H(v) \mathrm{d} x, \\
\mathrm{III} \rightarrow \int_{Q} H(v)\left|\partial_{t}\left(\eta^{q}\right)\right| \mathrm{d} z .
\end{gathered}
$$

For the term I, we have to be more careful. We begin by splitting

$$
\begin{aligned}
& \int_{Q} \delta_{h, j} A(\nabla u) \cdot \nabla\left(f\left(\left|\delta_{h} u\right|\right) \eta^{q} \delta_{h} u\right) \mathrm{d} z=\int_{Q} \delta_{h, j} A(\nabla u) \cdot\left(\nabla\left|\delta_{h} u\right|\right) f^{\prime}\left(\left|\delta_{h} u\right|\right) \eta^{q} \delta_{h, j} u \mathrm{~d} z \\
& \quad+f_{Q} \delta_{h, j} A(\nabla u) \cdot \delta_{h, j} \nabla u f\left(\left|\delta_{h} u\right|\right) \eta^{q} \mathrm{~d} z+f_{Q} \delta_{h} A(\nabla u) \cdot \nabla \eta q \eta^{q-1} f\left(\left|\delta_{h} u\right|\right) \delta_{h} u \mathrm{~d} z=: \mathrm{I}_{j}^{1}+\mathrm{I}_{j}^{2}+\mathrm{I}_{j}^{3}
\end{aligned}
$$


We get for $\mathrm{I}_{j}^{2}$ with Lemma 3.1

$$
\mathrm{I}_{j}^{2}=f_{Q} \delta_{h} A(\nabla u) \cdot \delta_{h, j} \nabla u f\left(\left|\delta_{h, j} u\right|\right) \eta^{q} \mathrm{~d} z \approx \int_{Q}\left|\delta_{h, j} V(\nabla u)\right|^{2} f\left(\left|\delta_{h, j} u\right|\right) \eta^{q} \mathrm{~d} z
$$

and because of $V(\nabla u) \in W^{1,2}(Q)$ we get as $h \rightarrow 0$

$$
\mathrm{I}_{j}^{2} \rightarrow f_{B}\left|\partial_{j} V(\nabla u)\right|^{2} f(v) \eta^{q} \mathrm{~d} z
$$

The estimate for $\mathrm{I}_{j}^{3}$ is of lower order. It can be treated in the same way as in the stationary case, see [13, Theorem 11]. The additional factor of $f(v)$ does not change the proof as every step is of pointwise manner. In explicit we find

$$
\limsup _{h \rightarrow 0} \sum_{j}\left|\mathrm{I}_{j}^{3}\right| \leq \delta f_{Q}|\nabla V(\nabla u)|^{2} f(v) \eta^{q} \mathrm{~d} z+c_{\delta} f_{Q}|V(\nabla u)|^{2}\|\nabla \eta\|_{\infty}^{2} f(v) \mathrm{d} z
$$

For $\mathrm{I}_{j}^{1}$ in (3.13), we note that $\left|\delta_{h} u\right| f^{\prime}\left(\left|\delta_{h} u\right|\right)$ is bounded uniformly in $h$ because of $f^{\prime}(t)=0$ for large $t$. For the integrand of $\mathrm{I}_{j}^{1}$ this gives with the help of Lemma 3.1

$$
\begin{aligned}
\left|\delta_{h} A(\nabla u) f^{\prime}\left(\left|\delta_{h} u\right|\right)\left(\nabla\left|\delta_{h} u\right|\right) \eta^{q} \delta_{h} u\right| & \leq\left|\delta_{h} A(\nabla u)\right||\nabla| \delta_{h} u||\left|f^{\prime}\left(\left|\delta_{h} u\right|\right) \delta_{h} u\right| \\
& \lesssim h^{-2}\left|\tau_{h} A(\nabla u)\right|\left|\tau_{h} \nabla u\right| \\
& \lesssim\left|\delta_{h} V(\nabla u)\right|^{2},
\end{aligned}
$$

where the constants depend on $f$. Since $\nabla V(\nabla u) \in L^{2}(Q)$, we can use the generalized theorem of dominated convergence and get a limit function in $L^{1}(Q)$. The next step is to proof that this term converges to something that is positive, for this we have to use the positivity of the second variation.

For the next steps we need the additional assumption that $\nabla^{2} u \in L^{s}(Q)$ for some $s>1$ and that $\varphi \in C^{2}([0, \infty))$. Later we explain how to remove this additional assumption. In this case, we know, that all quantities involving difference quotients have a well defined almost everywhere limit. We find

$$
\begin{aligned}
\sum_{j=1}^{n} \mathrm{I}_{j}^{1} & \rightarrow \sum_{j=1}^{n} f_{Q} \partial_{j} A(\nabla u) \cdot \nabla v f^{\prime}(v) \eta^{q} \partial_{j} u d z \\
& =\sum_{j, k=1}^{n} f_{Q} \partial_{j}\left(\frac{\varphi^{\prime}(v)}{v} \partial_{k} u\right) \partial_{k} v f^{\prime}(v) \eta^{q} \partial_{j} u d z \\
& =f_{Q}\left(\frac{\varphi^{\prime}(v)}{v}\left(|\nabla v|^{2}-\frac{|\nabla v \cdot \nabla u|^{2}}{v^{2}}\right)+\varphi^{\prime \prime}(v) \frac{|\nabla v \cdot \nabla u|^{2}}{v^{2}}\right) f^{\prime}(v) \mathrm{d} z
\end{aligned}
$$

using in the last step that $v \partial_{j} v=\sum_{k} \partial_{k} u \partial_{j} \partial_{k} u$.

Using the Cauchy-Schwartz inequality and the fact that $f^{\prime}(t) \geq 0$ we conclude that $\sum_{j=1}^{n} \mathrm{I}_{j}^{1}$ converges to a non-negative function and can be omitted.

Combining all limits the claim of the theorem follows, however under the additional assumption that $\nabla^{2} u \in L^{s}(Q)$ for same $s>1$ and $\varphi \in C^{2}([0, \infty))$.

Let us now explain how to overcome this additional assumption by means of an approximation argument. We proceed similar to [16] and [5. For this we approximate $\varphi$ by its shifted version $\varphi_{\lambda}$ for a small $\lambda>0$. In the end the limit $\lambda \searrow 0$ will imply the general result for $\varphi$. By $A_{\lambda}$ and $V_{\lambda}$ we denote the modified quantities $A$ and $V$.

We have $Q \Subset J \times \Omega$, so $J \times \Omega$ contains an enlarged $Q$. For the ease of presentation let us assume that $4 Q:=\left(t-4 \alpha R^{2}, t\right) \times 4 B \subset J \times \Omega$.

Due to (3.8) we have $u\left(t-2 \alpha R^{2}, \cdot\right) \in W^{1,2}(2 B)$. Now, for $\lambda>0$ small let $u_{\lambda}$ be the solutions of

$$
\begin{aligned}
\partial_{t} u_{\lambda}-\Delta_{\varphi_{\lambda}} u_{\lambda} & =0 \text { in } 2 Q \\
u_{\lambda} & =u \text { on } \partial_{\mathrm{par}}(2 Q) .
\end{aligned}
$$

Since $L^{\varphi_{\lambda}}(B)=L^{\varphi}(B)$, the existence of $u_{\lambda} \in L^{\infty}\left(L^{2}\right)$ with $\nabla u_{\lambda} \in L^{\varphi_{\lambda}}(2 Q)$ is standard. Moreover, by (3.7) we know that $\nabla V_{\lambda}\left(\nabla u_{\lambda}\right) \in L^{2}(Q)$ and $\nabla u_{\lambda} \in L^{\infty}\left(I, L^{2}(B)\right)$ uniformly in $\lambda>0$. 
Due to Lemma 4.3 of [16] it follows from $\nabla V_{\lambda}\left(\nabla u_{\lambda}\right) \in L^{2}(Q)$ that $\nabla^{2} u_{\lambda} \in L^{s}(Q)$ for some $s>1$, with $s$ independent of $\lambda$. This and $\varphi_{\lambda} \in C^{2}([0, \infty))$ implies that our calculations above are applicable to $u_{\lambda}$. In particular, we obtain that (3.9) is valid for $u$ replaced by $u_{\lambda}$ and $\varphi$ replaced by $\varphi_{\lambda}$. It remains to show the passage to the limit. For this is suffices to show that $\nabla u_{\lambda} \rightarrow \nabla u$ and $V_{\lambda}\left(\nabla u_{\lambda}\right) \rightarrow V(\nabla u)$ almost everywhere (for a subsequence) and $\nabla V_{\lambda}\left(\nabla u_{\lambda}\right) \rightarrow \nabla V(\nabla u)$ in $L^{2}(Q)$ as $\lambda \searrow 0$. This ensures the strong limit on the right hand side of (3.9) and by lower semicontinuity also on the left-hand side.

From the error we obtain

$$
\frac{1}{2}\left\|\left(u_{\lambda}-u\right)(t)\right\|_{2}^{2}+\int_{2 Q}\left(A_{\lambda}\left(\nabla u_{\lambda}\right)-A(\nabla u)\right) \cdot \nabla\left(u_{\lambda}-u\right) \mathrm{d} z=0 .
$$

Hence,

$$
\int_{2 Q}\left(A_{\lambda}\left(\nabla u_{\lambda}\right)-A_{\lambda}(\nabla u)\right) \cdot \nabla\left(u_{\lambda}-u\right) \mathrm{d} z \leq \int_{2 Q}\left(A(\nabla u)-A_{\lambda}(\nabla u)\right) \cdot \nabla\left(u_{\lambda}-u\right) \mathrm{d} z .
$$

It follows from Lemma 3.3 that

$$
\left|A_{\lambda}(Q)-A(Q)\right| \leq c \varphi_{|Q|}^{\prime}(\lambda) .
$$

The same argument applied to the Orlicz function $\psi_{|Q|}$ defined by $\psi_{|Q|}^{\prime}(t):=\sqrt{\varphi_{|Q|}^{\prime}(t) t}$ implies 1

$$
\left|V_{\lambda}(Q)-V(Q)\right|^{2} \leq c\left|\psi_{|Q|}^{\prime}(\lambda)\right|^{2} \approx c \varphi_{|Q|}(\lambda)
$$

Hence, from (3.20) we obtain with Lemma 3.1

$$
\left\|V_{\lambda}\left(\nabla u_{\lambda}\right)-V_{\lambda}(\nabla u)\right\|_{2}^{2} \lesssim \int_{2 Q} \varphi_{|\nabla u|}^{\prime}(\lambda)\left|\nabla\left(u_{\lambda}-u\right)\right| \mathrm{d} z
$$

Now Young's inequality with $\varphi_{|\nabla u|}$ and Lemma 3.1 imply

$$
\left\|V_{\lambda}\left(\nabla u_{\lambda}\right)-V_{\lambda}(\nabla u)\right\|_{2}^{2} \lesssim \int_{2 Q} \varphi|\nabla u|(\lambda) d z
$$

The right hand side convergence pointwise to zero for $\lambda \searrow 0$ and has the majorant $c(\varphi(|\nabla u|)+\varphi(\lambda)) \in$ $L^{1}(Q)$. This proves that

$$
\lim _{\lambda \searrow 0}\left\|V_{\lambda}\left(\nabla u_{\lambda}\right)-V_{\lambda}(\nabla u)\right\|_{2}^{2}=0 .
$$

It follows from (3.22) by the dominated convergence that $\left\|V_{\lambda}(\nabla u)-V(\nabla u)\right\|_{2}^{2} \rightarrow 0$. So $V_{\lambda}(\nabla u) \rightarrow$ $V(\nabla u)$ almost everywhere. Thus it follows from [16, Lemma 4.8] that also $\nabla u_{\lambda} \rightarrow \nabla u$ almost everywhere. This and the uniform boundedness of $\nabla V_{\lambda}\left(\nabla u_{\lambda}\right)$ in $L^{2}(Q)$ implies also $\nabla V_{\lambda}\left(\nabla u_{\lambda}\right) \rightarrow \nabla V(\nabla u)$ in $L^{2}(Q)$.

This completes the approximation argument and the proof is finished.

In the following we use the notation $(a)_{+}:=\max \{a, 0\}$ and $\{v>\gamma\}=\{(t, x): v(t, x)>\gamma\}$. We write $\chi_{A}$ for the indicator function of the set $A$.

We want to point out that Theorem 3.5 strongly simplify the proof in [10] for $p$-caloric functions, since we do not need to distinguish the sub-linear $p<2$ and super-linear $p>2$ case. In all situations we can choose the easy function $f(v)=\chi_{\{v>\gamma\}}$ in Theorem 3.5. This gives:

Corollary 3.6. Let $u, f, H$ be as in Theorem 3.5. For $\gamma>0$ let $G(t):=\left(\sqrt{\varphi^{\prime}(t) t}-\sqrt{\varphi^{\prime}(\gamma) \gamma}\right)_{+}$and $H(t)=\left(t^{2}-\gamma^{2}\right)_{+}$. Then we get

$$
\begin{aligned}
\sup _{I} & \frac{1}{\alpha R^{2}} \int_{B} H(v) \eta^{q} \mathrm{~d} x+f_{Q}\left|\nabla\left(G(v) \eta^{\frac{q}{2}}\right)\right|^{2} \mathrm{~d} z \\
& \lesssim f_{Q} \varphi(v)\|\nabla \eta\|_{\infty}^{2} \chi_{\{v>\gamma\}} \mathrm{d} z+f_{Q} H(v) \eta^{q-1}\left|\partial_{t} \eta\right| \mathrm{d} z .
\end{aligned}
$$

\footnotetext{
${ }^{1}$ Compare 13 for the use of $\psi$.
} 


\section{4. $L^{\infty}$-BOUNDS OF THE GRADIENT}

In this section we prove the boundedness of the gradients $\nabla u$ by means of the DiGeorgi technique. We assume that the assumptions of Theorem 3.5 are satisfied. In particular, $u$ is a local $\varphi$-caloric solution on $J \times \Omega$ and $Q=I \times B=\left(t-\alpha R^{2}, t\right) \times B_{R}(x) \Subset J \times \Omega$.

We define the sequence of scaled cylinders with the same center

$$
Q_{k}=2\left(1+2^{-k}\right) Q \text {. }
$$

Now choose $\zeta_{k} \in C_{0}^{\infty}\left(\mathbb{R}^{1+n}\right)$ with the following properties:

$$
\begin{aligned}
\chi_{Q_{k}} & \leq \zeta_{k} \leq \chi_{Q_{k+1}} \\
\left|\nabla \zeta_{k}\right| & \lesssim R^{-1} 2^{k} \\
\left|\partial_{t} \zeta_{k}\right| & \lesssim\left(\alpha R^{2}\right)^{-1} 2^{k} .
\end{aligned}
$$

For $\gamma_{\infty}>0$ (to be chosen later) we define

$$
\gamma_{k}:=\gamma_{\infty}\left(1-2^{-k}\right)
$$

For a function $f$ on $2 Q=2 I \times 2 B$ we define the following scaled Bochner type norms

$$
\|f\|_{L^{s}\left(L^{r}\right)(k)}:=\|\| f\left\|_{L^{s}\left(\zeta_{k}^{q} \mathrm{~d} x\right)}\right\|_{L^{r}(\mathrm{~d} t)}:=\left(f_{2 I}\left(f|f|^{r} \zeta_{k}^{q} \mathrm{~d} x\right)^{\frac{s}{r}} \mathrm{~d} t\right)^{\frac{1}{s}} .
$$

Recall that $v:=|\nabla u|$. For our DeGiorgi iteration argument we define the following important quantities

$$
\begin{aligned}
Y_{k} & :=\left\|\varphi(v) \chi_{\left\{v>\gamma_{k}\right\}}\right\|_{L^{1}\left(L^{1}\right)(k)} \\
Z_{k} & :=\frac{1}{\alpha}\left\|v^{2} \chi_{\left\{v>\gamma_{k}\right\}}\right\|_{L^{1}\left(L^{1}\right)(k)} \\
W_{k} & :=Y_{k}+Z_{k} .
\end{aligned}
$$

We start with some level set estimates in terms of $W_{k}$.

Lemma 4.1. Uniformly in $k$ we have

$$
\begin{aligned}
\frac{1}{\alpha}\left\|v^{2} \chi_{\left\{v>\gamma_{k+1}\right\}}\right\|_{L^{\infty}\left(L^{1}\right)(k+1)} & \lesssim 2^{3 k} W_{k} \\
\left\|\varphi(v) \chi_{\left\{v>\gamma_{k+1}\right\}}\right\|_{L^{1}\left(L^{\frac{n}{n-2}}\right)(k+1)} & \lesssim 2^{3 k} W_{k} .
\end{aligned}
$$

Proof. We define $G_{k}(t):=\left(\left(\varphi^{\prime}(t) t\right)^{\frac{1}{2}}-\left(\varphi^{\prime}\left(\gamma_{k}\right) \gamma_{k}\right)^{\frac{1}{2}}\right)_{+}$and $H(t)=\left(v^{2}-\gamma_{k}^{2}\right)_{+}$as above and recall the energy inequality from Corollary 3.6 with $\eta=\left(\zeta_{k+1}^{\frac{n-2}{n}}\right)$ :

$$
\begin{aligned}
& \sup _{I} \frac{1}{\alpha} \int_{B} H_{k}(v) \zeta_{k+1}^{q \frac{n}{n-2}} \mathrm{~d} x+R^{2} f_{Q}\left|\nabla\left(G_{k}(v) \zeta_{k+1}^{\frac{q}{2} \frac{n}{n-2}}\right)\right|^{2} \mathrm{~d} z \\
& \quad \lesssim R^{2} f_{Q} \varphi(v)\left\|\nabla\left(\zeta_{k+1}^{\frac{n}{n-2}}\right)\right\|_{\infty}^{2} \chi_{\left\{v>\gamma_{k+1}\right\}} \mathrm{d} z+R^{2} f_{Q} H(v) \zeta_{k+1}^{(q-1) \frac{n}{n-2}} \partial_{t}\left(\zeta^{\frac{n}{n-2}}\right) \mathrm{d} z
\end{aligned}
$$

At first we estimate the terms on the right hand side of 4.3 and note that $\zeta_{k} \equiv 1$ on $\operatorname{supp} \zeta_{k+1}$ :

$$
\begin{aligned}
R_{Q}^{2} \int_{Q} \varphi(v) \chi_{\left\{v>\gamma_{k}\right\}}\left\|\nabla\left(\zeta_{k+1}^{\frac{n}{n-2}}\right)\right\|_{\infty}^{2} \mathrm{~d} z & \lesssim 2^{2 k} f_{Q} \varphi(v) \chi_{\left\{v>\gamma_{k}\right\}} \chi_{\operatorname{supp} \chi_{\{k+1\}}} \mathrm{d} z \\
& \leq 2^{2 k} f_{Q} \varphi(v) \chi_{\left\{v>\gamma_{k}\right\}} \zeta_{k}^{q} \mathrm{~d} z \\
& =2^{2 k} Y_{k}
\end{aligned}
$$


and

$$
\begin{aligned}
R^{2} f_{Q} H_{k}(v)\left(\zeta_{k+1}^{\frac{n-2}{n}}\right)^{q-1}\left|\partial_{t}\left(\zeta_{k+1}^{\frac{n-2}{n}}\right)\right| \mathrm{d} z & \lesssim \frac{2^{k+1} R^{2}}{\alpha R^{2}} f_{Q} v^{2} \chi_{\left\{v>\gamma_{k}\right\}} \chi_{\operatorname{supp} \chi_{\{k+1\}}} \mathrm{d} z \\
& \lesssim \frac{2^{k}}{\alpha} f_{Q} v^{2} \chi_{\left\{v>\gamma_{k}\right\}} \zeta_{k}^{q} \mathrm{~d} z \\
& =2^{k} Z_{k} \leq 2^{2 k} Z_{k}
\end{aligned}
$$

Putting this in 4.3 gives

$$
\sup _{I} \frac{1}{\alpha} \int_{B} H_{k}(v) \zeta_{k+1}^{q \frac{n}{n-2}} \mathrm{~d} x+R^{2} f_{Q}\left|\nabla\left(G_{k}(v) \zeta_{k+1}^{\frac{q}{2} \frac{n}{n-2}}\right)\right|^{2} \mathrm{~d} z \lesssim 2^{2 k} W_{k}
$$

To prove 4.1 we first note that for $h(t)=t^{2}$ or $h(t)=\left(\varphi^{\prime}(t) t\right)^{\frac{1}{2}}$ we get:

$$
\begin{aligned}
h(v) & =h(v)-h\left(\gamma_{k}\right)+h\left(\gamma_{k}\right) \\
& =h(v)-h\left(\gamma_{k}\right)+\frac{h\left(\gamma_{k}\right)}{h\left(\gamma_{k+1}\right)-h\left(\gamma_{k}\right)}\left(h\left(\gamma_{k+1}\right)-h\left(\gamma_{k}\right)\right) \\
& \leq\left(h(v)-h\left(\gamma_{k}\right)\right) \frac{h\left(\gamma_{k+1}\right)}{h\left(\gamma_{k+1}\right)-h\left(\gamma_{k}\right)} \\
& \leq \frac{h\left(\gamma_{k+1}\right)}{h\left(\gamma_{k+1}\right)-h\left(\gamma_{k}\right)}\left(h(v)-h\left(\gamma_{k}\right)\right)_{+}
\end{aligned}
$$

and for $k \geq 1$ we get using the intermediate value theorem of differential calculus with some $t \in$ $\left(\gamma_{k}, \gamma_{k+1}\right)$ and the fact that $h(2 t) \lesssim h(t)$ and $t h^{\prime}(t) \approx h(t)$ :

$$
\frac{h\left(\gamma_{k+1}\right)}{h\left(\gamma_{k+1}\right)-h\left(\gamma_{k}\right)}=\frac{h\left(\gamma_{k+1}\right)}{h^{\prime}(t)\left(\gamma_{k+1}-\gamma_{k}\right)} \approx \frac{h\left(\gamma_{k+1}\right) t}{h(t)\left(c\left(2^{-k}-2^{-k-1}\right)\right)} \lesssim \frac{h\left(\gamma_{k+1}\right)}{h\left(\frac{\gamma_{k+1}}{2}\right)} 2^{k+1} \lesssim 2^{k+1}
$$

So in total we have

$$
h(v) \chi_{\left\{v>\gamma_{k+1}\right\}} \lesssim 2^{k+1}\left(h(v)-h\left(\gamma_{k}\right)\right)_{+}
$$

and we see that $\zeta \leq \zeta^{\frac{n-2}{n}}$ as $0 \leq \zeta \leq 1$. Putting this in 4.4 gives

$$
\begin{aligned}
\left\|v^{2} \chi_{\left\{v>\gamma_{k+1}\right\}}\right\|_{L^{\infty}\left(L^{1}\right)(k+1)} & =\alpha \sup _{I} \frac{1}{\alpha} f_{B} v^{2} \chi_{\left\{v>\gamma_{k+1}\right\}} \zeta_{k+1}^{q} \mathrm{~d} x \\
& \lesssim \alpha 2^{k} \sup _{I} \frac{1}{\alpha} f_{B} H_{k}(v)\left(\zeta_{k+1}^{\frac{n-2}{n}}\right)^{q} \mathrm{~d} x \\
& \lesssim \alpha 2^{3 k} W_{k}
\end{aligned}
$$

For inequality 4.2 we set $h(t)=\left(\varphi^{\prime}(t) t\right)^{\frac{1}{2}}$ in 4.5 and get $\varphi(t)^{\frac{1}{2}} \chi_{t>\gamma_{k+1}} \sim\left(\varphi^{\prime}(t) t\right)^{\frac{1}{2}} \chi_{t>\gamma_{k+1}} \lesssim 2^{k} G_{k}(t)$ for $t>\gamma_{k+1}$. We use Sobolev's embedding inequality and the previous estimates to find

$$
\begin{aligned}
\left\|\varphi(v) \chi_{\left\{v>\gamma_{k+1}\right\}}\right\|_{L^{1}\left(L^{\frac{n}{n-2}}\right)(k+1)} & =\|\| \varphi(v) \chi_{\left\{v>\gamma_{k+1}\right\}} \zeta_{k+1}^{q \frac{n-2}{n}}\left\|_{L^{\frac{n}{n-2}}(\mathrm{~d} x)}\right\|_{L^{1}(\mathrm{~d} t)} \\
& =\|\| \varphi(v)^{\frac{1}{2}} \chi_{\left\{v>\gamma_{k+1}\right\}} \zeta_{k+1}^{\frac{q}{2} \frac{n-2}{n}}\left\|_{L^{\frac{2 n}{n-2}}(\mathrm{~d} x)}^{2}\right\|_{L^{1}(\mathrm{~d} t)} \\
& \lesssim 2^{k}\|\| G_{k}(v) \zeta_{k+1}^{\frac{q}{2} \frac{n-2}{n}}\left\|_{L^{\frac{2 n}{n-2}}(\mathrm{~d} x)}^{2}\right\|_{L^{1}(\mathrm{~d} t)} \\
& \lesssim 2^{k} R^{2}\|\| \nabla\left(G_{k}(v) \zeta^{\frac{q}{2} \frac{n-2}{n}}\right)\left\|_{L^{2}(\mathrm{~d} x)}^{2}\right\|_{L^{1}(\mathrm{~d} t)} \\
& =2^{k} R^{2} f\left|\nabla\left(G_{k}(v) \zeta^{\frac{q}{2} \frac{n-2}{n}}\right)\right|^{2} \mathrm{~d} z \\
& \lesssim 2^{3 k} W_{k}
\end{aligned}
$$


This concludes the proof of the lemma.

We are now able to proof the main Theorem 2.2.

Proof of Theorem 2.2. We use the definitions from Lemma4.1 For $Y_{k+1}$ we get, by Hölder's inequality, for the couple $\left(\frac{n}{n-2}, \frac{n}{2}\right)$ (for $n \geq 3$ )

$$
\begin{aligned}
Y_{k+1} & =\left\|\varphi(v) \chi_{\left\{v>\gamma_{k+1}\right\}}\right\|_{L^{1}\left(L^{1}\right)(k+1)}=\left\|\frac{v^{\frac{4}{n}}}{v^{\frac{4}{n}}} \varphi(v) \chi_{\left\{v>\gamma_{k+1}\right\}}\right\|_{L^{1}\left(L^{1}\right)(k+1)} \\
& \leq \frac{1}{\gamma_{k+1}^{\frac{4}{n}}}\left\|\varphi(v) v^{\frac{4}{n}} \chi_{\left\{v>\gamma_{k+1}\right\}}\right\|_{L^{1}\left(L^{1}\right)(k+1)} \\
& \lesssim \frac{1}{\gamma_{\infty}^{\frac{4}{n}}}\left\|\varphi(v) \chi_{\left\{v>\gamma_{k+1}\right\}}\right\|_{L^{1}\left(L^{\frac{n}{n-2}}\right)(k+1)}\left\|v^{\frac{4}{n}} \chi_{\left\{v>\gamma_{k+1}\right\}}\right\|_{L^{\infty}\left(L^{\frac{n}{2}}\right)(k+1)} \\
& =\frac{1}{\gamma_{\infty}^{\frac{4}{n}}}\left\|\varphi(v) \chi_{\left\{v>\gamma_{k+1}\right\}}\right\|_{L^{1}\left(L^{\frac{n}{n-2}}\right)(k+1)}\left\|v^{2} \chi_{\left\{v>\gamma_{k+1}\right\}}\right\|_{L^{\infty}\left(L^{1}\right)(k+1)}^{\frac{2}{n}} \\
& \lesssim 2^{3 k\left(1+\frac{2}{n}\right)} W_{k}\left(\frac{W_{k} \alpha}{\gamma_{\infty}^{2}}\right)^{\frac{2}{n}} .
\end{aligned}
$$

(Note that for $n=1,2$ we can use any couple $\left(q, q^{\prime}\right)$.) And now for $Z_{k+1}$, we use the function $\rho(t):=\varphi(t) t^{\frac{4}{n}-2}$ and estimate

$$
\begin{aligned}
\alpha Z_{k+1} & =\left\|v^{2} \chi_{\left\{v>\gamma_{k+1}\right\}}\right\|_{L^{1}\left(L^{1}\right)(k+1)}=\left\|\frac{\rho(v)^{\frac{2}{n}}}{\rho(v)^{\frac{2}{n}}} v^{2} \chi_{\left\{v>\gamma_{k+1}\right\}}\right\|_{L^{1}\left(L^{1}\right)(k+1)} \\
& \leq \frac{1}{\rho\left(\gamma_{k+1}\right)^{\frac{2}{n}}}\left\|\varphi(v) v^{\frac{4}{n}} \chi_{\left\{v>\gamma_{k+1}\right\}}\right\|_{L^{1}\left(L^{1}\right)(k+1)} \\
& \lesssim \frac{1}{\rho\left(\gamma_{\infty}\right)^{\frac{2}{n}}}\left\|\varphi(v) \chi_{\left\{v>\gamma_{k+1}\right\}}\right\|_{L^{1}\left(L^{\frac{n}{n-2}}\right)(k+1)}\left\|v^{\frac{4}{n}} \chi_{\left\{v>\gamma_{k+1}\right\}}\right\|_{L^{\infty}\left(L^{\frac{2}{n}}\right)(k+1)} \\
& =\frac{1}{\rho\left(\gamma_{\infty}\right)^{\frac{2}{n}}}\left\|\varphi(v) \chi_{\left\{v>\gamma_{k+1}\right\}}\right\|_{L^{1}\left(L^{\frac{n}{n-2}}\right)(k+1)}\left\|v^{2} \chi_{\left\{v>\gamma_{k+1}\right\}}\right\|_{L^{\infty}\left(L^{1}\right)(k+1)}^{\frac{2}{n}} \\
& \lesssim 2^{3 k\left(1+\frac{2}{n}\right)} W_{k}\left(\frac{W_{k} \alpha}{\rho\left(\gamma_{\infty}\right)}\right)^{\frac{2}{n}} .
\end{aligned}
$$

In total, we have

$$
\begin{aligned}
W_{k+1} & =Y_{k+1}+Z_{k+1} \\
& \lesssim 2^{3 k\left(1+\frac{2}{n}\right)} W_{k}\left(\frac{W_{k} \alpha}{\gamma_{\infty}^{2}}\right)^{\frac{2}{n}}+2^{3 k\left(1+\frac{2}{n}\right)} \frac{W_{k}}{\alpha}\left(\frac{W_{k} \alpha}{\rho\left(\gamma_{\infty}\right)}\right)^{\frac{2}{n}} \\
& \lesssim 2^{3 k\left(1+\frac{2}{n}\right)} W_{k} \max \left\{\left(\frac{W_{k} \alpha}{\gamma_{\infty}^{2}}\right)^{\frac{2}{n}},\left(\frac{W_{k} \alpha^{\frac{2-n}{n}}}{\rho\left(\gamma_{\infty}\right)}\right)^{\frac{2}{n}}\right\} \\
& =2^{3 k\left(1+\frac{2}{n}\right)} W_{k}\left(\frac{W_{k}}{\min \left\{\frac{\rho\left(\gamma_{\infty}\right)}{\alpha^{\frac{2-n}{2}}}, \frac{\gamma_{\infty}^{2}}{\alpha}\right\}}\right)^{\frac{2}{n}}
\end{aligned}
$$

and the theorem follows from Lemma 4.1 in [10] as we have $W_{k} \rightarrow 0$ if we choose $\gamma_{\infty}$ such that $W_{0} \approx \min \left\{\frac{\rho\left(\gamma_{\infty}\right)}{\alpha \frac{2-n}{2}}, \frac{\gamma_{\infty}^{2}}{\alpha}\right\}$. This implies

$$
\min \left\{\frac{\rho(v)}{\alpha^{\frac{2-n}{2}}}, \frac{v^{2}}{\alpha}\right\} \leq \min \left\{\frac{\rho\left(\gamma_{\infty}\right)}{\alpha^{\frac{2-n}{2}}}, \frac{\gamma_{\infty}^{2}}{\alpha}\right\} \approx W_{0}=f_{Q} \varphi(v)+\frac{v^{2}}{\alpha} \mathrm{d} z
$$

This proves our main theorem. 


\section{REFERENCES}

[1] E. Acerbi and G. Mingione. Gradient estimates for a class of parabolic systems. Duke Math. J., 136(2):285-320, 2007.

[2] P. Baroni. Riesz potential estimates for a general class of quasilinear equations. Calc. Var. Partial Differential Equations, 53(3-4):803-846, 2015.

[3] P. Baroni and C. Lindfors, The Cauchy-Dirichlet problem for a general class of parabolic equations, Ann. Inst. H. Poincaré Anal. Non Linéaire 34 (2017), no. 3, 593-624.

[4] L. Belenki, L. Diening, and C. Kreuzer. Optimality of an adaptive finite element method for the $p$-Laplacian equation. IMA J. Numer. Anal., 32(2):484-510, 2012.

[5] L. C. Berselli, L. Diening, and M. Růžička, Existence of strong solutions for incompressible fluids with shear dependent viscosities, J. Math. Fluid Mech. 12 (2010), no. 1, 101-132.

[6] M. Bildhauer. Convex variational problems, volume 1818 of Lecture Notes in Mathematics. Springer-Verlag, Berlin, 2003.

[7] V. Bögelein, F. Duzaar, and G. Mingione. The regularity of general parabolic systems with degenerate diffusion. Mem. Amer. Math. Soc., 221(1041):vi-143, 2013.

[8] D. Breit, B. Stroffolini, and A. Verde. A general regularity theorem for functionals with $\varphi$-growth. Journal of Mathematical Analysis and Applications, 383(1):226-233, 2011.

[9] L. A. Caffarelli and I. Peral. On $W^{1, p}$ estimates for elliptic equations in divergence form. Comm. Pure Appl. Math., 51(1):1-21, 1998.

[10] E. DiBenedetto. Degenerate parabolic equations. Springer-Verlag, New York, 1993.

[11] E. DiBenedetto and A. Friedman. Regularity of solutions of nonlinear degenerate parabolic systems. J. reine angew. Math, 349:83-128, 1984.

[12] E. DiBenedetto and A. Friedman. Hölder estimates for nonlinear degenerate parabolic systems. J. Reine Angew. Math., 357:1-22, 1985.

[13] L. Diening and F. Ettwein. Fractional estimates for non-differentiable elliptic systems with general growth. Forum Mathematicum, 20(3):523-556, 2008.

[14] L. Diening, P. Kaplický, and S. Schwarzacher. BMO estimates for the p-Laplacian. Nonlinear Anal., 75(2):637-650, 2012.

[15] L. Diening and C. Kreuzer. Linear convergence of an adaptive finite element method for the $p$-Laplacian equation. SIAM J. Numer. Anal., 46:614-638, 2008.

[16] L. Diening, B. Stroffolini, and A. Verde, Everywhere regularity of functionals with $\varphi$-growth, Manuscripta Math. 129 (2009), no. 4, 449-481.

[17] L. Diening, B. Stroffolini, and A. Verde. The $\varphi$-harmonic approximation and the regularity of $\varphi$-harmonic maps. J. Differential Equations, 7:1943-1958, 2012.

[FS15] J. Frehse and S. Schwarzacher, On regularity of the time derivative for degenerate parabolic systems, SIAM J. Math. Anal. 47 (2015), no. 5, 3917-3943.

[18] T. Iwaniec. On $L^{p}$-integrability in PDE's and quasiregular mappings for large exponents. Annales Academice Scientiarum Fennica, Series A. I. Mathematica, 7:301-322, 1982.

[19] T. Kuusi and G. Mingione. Linear potentials in nonlinear potential theory. Arch. Ration. Mech. Anal., 207(1):215246,2013

[20] T. Kuusi and G. Mingione. Riesz potentials and nonlinear parabolic equations. Arch. Ration. Mech. Anal., $212(3): 727-780,2014$.

[21] G. M. Lieberman. The natural generalization of the natural conditions of Ladyzhenskaya and Ural' tseva for elliptic equations. Comm. Partial Differential Equations, 16(2-3):311-361, 1991.

[22] G. M. Lieberman. Hölder regularity for the gradients of solutions of degenerate parabolic systems. Ukr. Math. Bull, 3:352-373, 2006.

[23] P. Marcellini and G. Papi. Nonlinear elliptic systems with general growth. J. Differential Equations, 221(2):412-443, 2006.

[24] M. Misawa. Local Hölder regularity of gradients for evolutional p-Laplacian systems. Ann. Mat. Pura Appl. (4), 181(4):389-405, 2002.

[25] J. Moser. A Harnack inequality for parabolic differential equations. Comm. Pure Appl. Math., 17:101-134, 1964.

[26] J. Nash. Continuity of solutions of parabolic and elliptic equations. American Journal of Mathematics, pages 931-954, 1958

[27] S. Schwarzacher. Hölder-Zygmund estimates for degenerate parabolic systems. Journal of Differential Equations, 256:2423-2448, 2014.

[28] K. Uhlenbeck. Regularity for a class of non-linear elliptic systems. Acta Math., 138(3-4):219-240, 1977.

[29] N. N. Ural'ceva. Degenerate quasilinear elliptic systems. Zap. Naučn. Sem. Leningrad. Otdel. Mat. Inst. Steklov. (LOMI), 7:184-222, 1968

Bielefeld University, Faculty of Mathematics, Germany

E-mail address: lars.diening@uni-bielefeld.de

Mathematical Institute, OX2 6GG, University of Oxford

E-mail address: toni.scharle@maths.ox.ac.uk

Institute of Applied Mathematics, Bonn University, Germany \& Department of Mathematical Analysis, Charles University, Prague, Czech Republic

E-mail address: schwarz@karlin.mff.cuni.cz 